\title{
Neutrophil activation by immune complexes and the role of rheumatoid factor
}

\author{
R. GALE, J. V. BERTOUCH, T. P. GORDON, J. B RADLEY, AND \\ P. J. ROBERTS-THOMSON
}

From the Department of Clinical Immunology, Flinders Medical Centre, Bedford Park, South Australia 5042

SUMMARY Membrane activation of human neutrophils by preformed immune complexes and heat aggregated human gammaglobulins was studied by chemiluminescence. Strong neutrophil activation was found with human-albumin rabbit-antialbumin complexes prepared at equivalence, with maximal activation occurring in slight antigen excess. Furthermore different preparations of heat aggregated gammaglobulin which were of large size also showed similar activity. In contrast, heat aggregates of small size were inactive and blocked the chemiluminescent response found with larger active aggregates. A purified monoclonal rheumatoid factor with specificity for IgG modulated these responses when preincubated with preformed complexes or aggregates. Both enhancement of the neutrophil chemiluminescence response with inactive preparations and suppression of the response with highly active preparations were observed. Kinetic studies of the neutrophil chemiluminescent response varied with respect to the activating preparation, but were generally biphasic. This observation suggested an initial direct membrane activation followed by a more delayed response reflecting phagocytosis of complexes. We have demonstrated the direct activation of neutrophil chemiluminescence by laboratory preparations of immune complexes. The chemiluminescent responses observed were influenced by both the size and immunochemical properties of the activating complexes and by the presence of rheumatoid factor. These observations may have important implications in the immunopathogenesis of immune-complex-mediated diseases.

The activation of neutrophils by the interaction of soluble and insoluble material with neutrophil surface receptors leads to the release of oxygen-free radicals and lysosomal enzymes. ${ }^{1}$ The localised release of these toxic substances could bring about tissue inflammation, and such a mechanism has been strongly implicated in the immunopathogenesis of rheumatoid arthritis. ${ }^{1-3}$

We have previously reported the direct activation of neutrophils by sera and synovial fluid obtained from patients with rheumatoid arthritis and Felty's syndrome. ${ }^{4}$ Similar results have been reported by Starkebaum et al. ${ }^{1}$ In both studies neutrophil activation was assessed by chemiluminescence (CL). In our study we obtained evidence which suggested that the activating material consisted of large-molecularweight immune complexes containing IgG. However,

Accepted for publication 11 January 1983.

Correspondence to Dr P. Roberts-Thomson, Department of Clinical Immunology, Flinders Medical Centre, Bedford Park, South Australia 5042. further characterisation of these complexes proved difficult, and we could not demonstrate a significant correlation between neutrophil activation induced by these fluids and 2 different assays for immune complexes. These results suggested that the immune complexes differed in immunochemical and biological properties, with a subgroup having the important property of activating neutrophils.

We have therefore examined the properties of immune complexes which activate neutrophils in vitro and have further investigated the modulation of these responses by a purified monoclonal rheumatoid factor. This reagent has provided a means of investigating the possible role that anti-immunoglobulins play in the immunopathogenesis of rheumatoid arthritis.

\section{Materials and methods}

Neutrophils. These were prepared from heparinised blood obtained from group $\mathrm{O}$ healthy individuals. 
The blood was diluted with an equal volume of Dulbecco's phosphate buffered saline (PBS) and the mononuclear cells removed by centrifuging through Ficollpaque (Pharmacia). The pellet was resuspended in twice the volume of $2 \%$ dextran in PBS and allowed to stand at room temperature for 45 minutes to sediment the red cells. The supernatant containing the neutrophils was removed, and contaminating red cells were lysed during a 5-minute incubation in Gey's solution. The preparation was adjusted to $5 \times 10^{6}$ neutrophils $/ \mathrm{ml}\left(5 \times 10^{9} / \mathrm{l}\right)$ in modified Eagle's minimal essential medium (MEM, Flow Laboratories) containing $1 \%$ fetal calf serum and maintained at $37^{\circ} \mathrm{C}$.

Luminol. The stock solution was $0.056 \mathrm{M}$ 3-aminophthalhydrazide (Koch Light Laboratories) in dimethyl sulphoxide. This was diluted 1:40 in MEM and prewarmed to $37^{\circ} \mathrm{C}$ before use.

Chemiluminescence. CL was measured in an LKB luminometer (Model 1250) fitted with a $37^{\circ} \mathrm{C}$ waterjacketed sample holder and low-speed rotation of the reaction vessel. Either of the 2 following methods of CL measurement were employed.

(a) Chemiluminescence maximum (CL max). 200 $\mu \mathrm{l}$ of neutrophil preparation was incubated at $37^{\circ} \mathrm{C}$ for 5 minutes with $100 \mu$ l of the test preparation. 600 $\mu$ l of luminol solution was then added and the $\mathrm{CL}$ activity recorded. The CL max was defined as the highest recorded light output (in millivolts) integrated over 1-second intervals.

(b) Chemiluminescent kinetic studies. $100 \mu \mathrm{l}$ of the test preparation were mixed with $600 \mu \mathrm{l}$ of luminol solution and $200 \mu$ l of the neutrophil preparation and immediately placed in the luminometer. The rate of $\mathrm{CL}$ was measured for the following 5 minutes, by integration over 10 -second intervals.

Heat aggregated gammaglobulin (HAGG). Cohn fraction 11 gammaglobulin $(160 \mathrm{mg} / \mathrm{ml},(160 \mathrm{~g} / \mathrm{l})$, Commonwealth Serum Laboratories) was diluted $1: 8$ with PBS. This was aggregated by heating at $63^{\circ} \mathrm{C}$ for $15,30,45,60,75$, or $90 \mathrm{~min}$.

Preincubation with monoclonal rheumatoid factor $(m R F)$. Preparations of HAGG or preformed immune complexes were preincubated with $10 \% \mathrm{v} / \mathrm{v}$ solution of a purified preparation of $\operatorname{mRF}(8 \cdot 6 \mathrm{~g} / \mathrm{l})$ for 60 minutes at room temperature before testing for neutrophil CL activity. The $\mathrm{mRF}$ was obtained from a patient with a lymphoproliferative disorder and its purification and properties have been previously described. $^{5}$

Filtration chromatography. Gel filtration was performed at room temperature by means of a $90 \times 2.5$ cm Sephacryl S300 (Pharmacia) column with PBS without preservative as eluant. An upward flow rate of $20 \mathrm{ml} /$ hour was maintained and $6 \mathrm{ml}$ fractions were collected.
HAGG centrifugation. HAGG, prepared by heating for 75 minutes, was centrifuged at $8000 \mathrm{~g}$ on a Beckman Microfuge (Model B). The supernate was aspirated and the deposit reconstituted to the original volume with PBS.

Preformed immune complexes. Purified human albumin (Sigma) was incubated with rabbit antialbumin (Dako) in PBS at room temperature for one hour. Albumin concentrations ranging from 1 to 50 $\mu \mathrm{g}$ in $1 \mathrm{ml}$ were incubated with $20 \mu \mathrm{l}$ of antisera. Immune complex precipitation as reflected by the degree of light scatter (in millivolts) was measured with a Behring laser nephelometer.

\section{Results}

Samples of HAGG were prepared by heating at $63^{\circ} \mathrm{C}$ for periods ranging from 15 to 90 minutes. Each preparation was tested for neutrophil activation by measuring the CL max. As shown in Fig. 1, heating for more than 45 minutes was required to produce aggregates which activated neutrophil CL.

Preincubation of these preparations with $\mathrm{mRF}$ altered these responses by inducing activity in previously inactive preparations and suppressing the peak response seen in highly reactive preparations (Fig. 1).



Fig. 1 The neutrophil CL max values observed following activation with HAGG preparations prepared by heating for varying time periods. Normal activation activation following preincubation of the HAGG with $m R F--1$. 


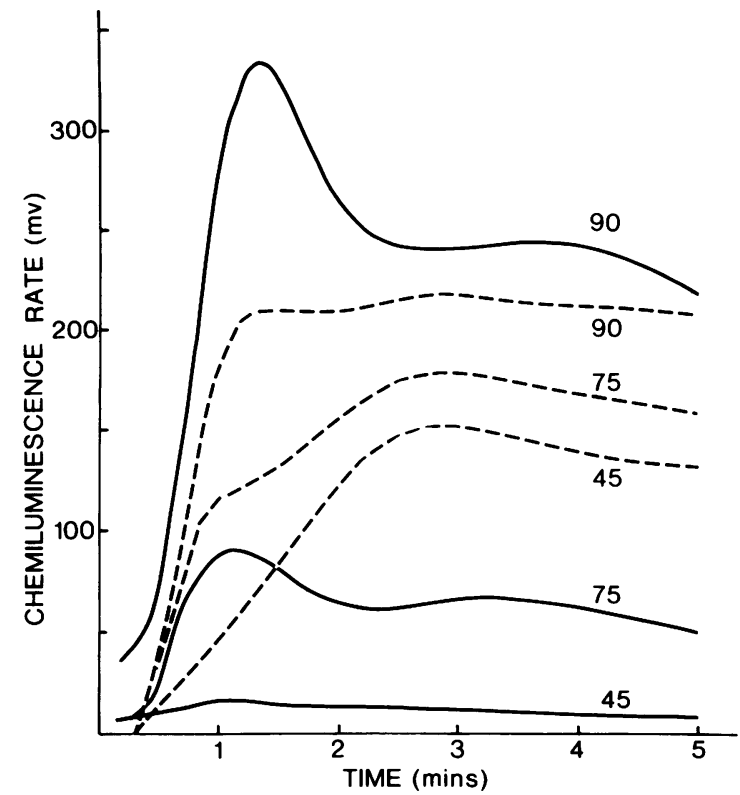

Fig. 2 The rate of neutrophil CL during 5 minutes incubation with 3 different preparations of $H A G G$ which had been prepared by heating for 45, 75, and 90 minutes respectively. Activation by the same 3 preparations following preincubation with $m R F$ are shown as - - -

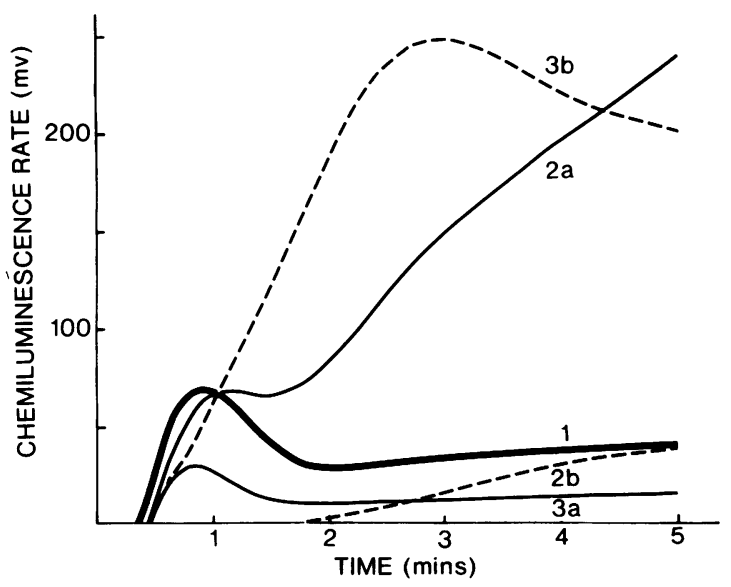

Fig. 3 The rate of neutrophil $C L$ during the first 5 minutes of incubation with HAGG prepared by heating for 75 minutes and the deposit and supernate after centrifugation of the same preparation. (1) Original HAGG preparation; (2a) deposit; (2b) deposit preincubated with $\mathrm{mRF}$; $(3 a)$ supernate; $(3 b)$ supernate preincubated with $m R F$.

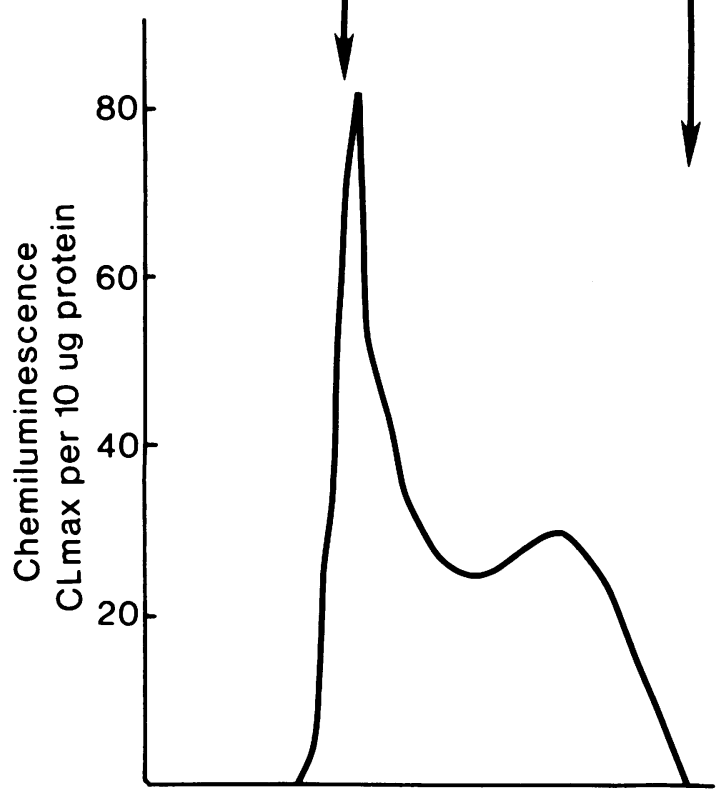

\section{Elution volume}

Fig. 4 The neutrophil CL max values for the eluate fractions of a HAGG preparation separated by column chromatography and adjusted to the same protein concentration. The positions of the void volume (Vo) and monomeric $\mathrm{IgG}$ in relation to elution volume are shown.

These observations were supported by kinetic studies as seen in Fig. 2, where neutrophil CL responses were observed with different samples of HAGG in the presence and absence of $\mathrm{mRF}$. With HAGG alone a biphasic response was seen, with an initial peak occurring within the first $60-90$ seconds and a slower second response taking 3 minutes or longer to reach its peak.

Two preparations of HAGG were examined in more detail by separating aggregates according to molecular size either by centrifugation or by filtration chromatography. An active preparation was centrifuged and the precipitate and supernate, both with and without preincubation with $\mathrm{mRF}$, were tested for neutrophil CL (Fig. 3). Almost all the active material of the preparation was found in the centrifuged deposit. Preincubation with the $\mathrm{mRF}$ caused a depression in the activity of the deposit and enhanced that of the previously poorly active supernatant.

An inactive preparation of HAGG (heated for 30 min) was separated by filtration chromatography and the column eluate fractions diluted if necessary to contain a uniform $100 \mu \mathrm{g} / \mathrm{ml}(\mathrm{mg} / \mathrm{l})$ of protein. These 


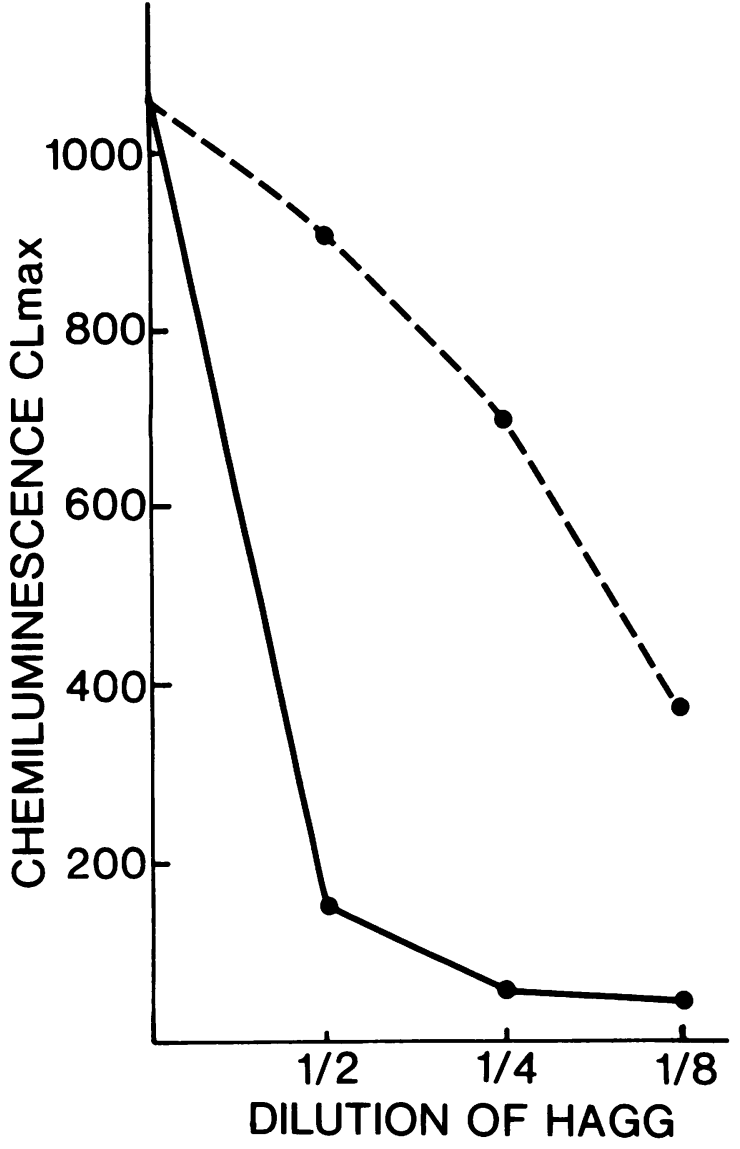

Fig. 5 The neutrophil CL max values obtained with a neutrophil activating preparation of $H A G G$ diluted in parallel with $P B S---$ and a nonactivating preparation of $H A G G$

column fractions were then tested for neutrophil activation. As shown in Fig. 4, the aggregates eluting in the void volume, and therefore greater than $10^{6}$ Daltons in molecular size, showed activity. The unseparated preparation continued to remain inactive.

Dilution of an active HAGG preparation with an inactive HAGG preparation as diluent showed a marked depression of the response relative to an equivalent dilution with PBS alone (Fig. 5).

An equivalence curve of preformed immune complexes of human albumin and rabbit antialbumin was prepared and the zone of equivalence determined nephelometrically (Fig. 6). Antigen/antibody equivalence was determined to be between 15-30 $\mu \mathrm{g}$ albumin with $20 \mu$ l of antiserum and this was subsequently confirmed by a radiolabelled precipitin curve

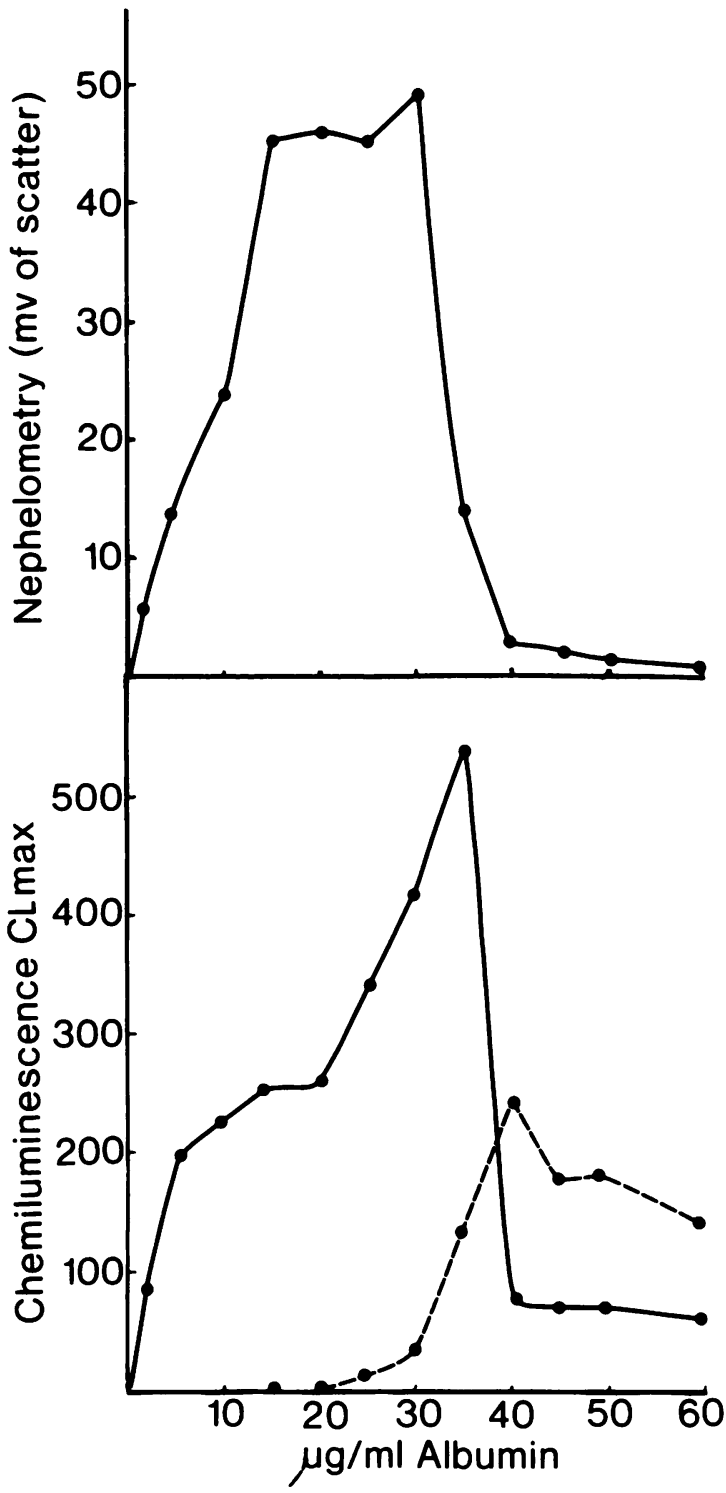

Fig. 6 The nephelometric values for an equivalence curve of immune complexes containing human-albumin/rabbit antihuman-albumin. The concentration of albumin mixed with a fixed $20 \mu$ lof antialbumin is shown. The neutrophil $C L$ max values with these preparations are shown and the CL max after preincubation of the complexes with $m R F--$.

(data not shown). Neutrophil CL was observed with immune complexes prepared near antigen/antibody equivalence, with maximal activation occurring with preparations containing $30-35 \mu \mathrm{g}$ albumin, which 
was in the region of slight antigen excess. The modulation of these responses by preincubation of the complexes with $\mathrm{mRF}$ again showed both depression of the neutrophil CL response with large active complexes and the enhancement of activation with small, previously poorly active complexes.

\section{Discussion}

Recent studies of the immune complexes detected in association with various autoimmune diseases have suggested that the size and immunochemical characteristics of the complexes may be important in determining pathogenicity. ${ }^{6}$ In this study supporting evidence was obtained for this hypothesis by using preparations of HAGG and preformed immune complexes of varying sizes to activate neutrophils. Activation of neutrophils was measured by CL.

The longer the period of heating at $63^{\circ} \mathrm{C}$ used in the preparation of HAGG the larger and less soluble the aggregates. ${ }^{8}$ Heating for longer than 45 minutes appeared necessary to prepare aggregates which induced CL in our study. However, these HAGG preparations contained a mixture of aggregate sizes, and removal of the small inactive aggregates increased the neutrophil response to the remaining larger active aggregates. Our observations suggested an initial blocking of neutrophil $\mathrm{Fc}$ membrane receptors by smaller inactive aggregates.

The biphasic neutrophil CL response to HAGG observed in the 5-minute kinetic studies may represent a rapid membrane-associated response followed by a slower phagocytosis-dependent emission. This differentiation of 2 separate mechanisms of CL response has been reported. ${ }^{9}$ The release of oxygenfree radicals independent of phagocytosis by means of cytochalasin-B-treated neutrophils was observed by Goldstein et al., ${ }^{10}$ and we intend to examine this activation mechanism further with our model.

Preincubation of HAGG with mRF produced both enhancement and depression in the subsequent neutrophil CL responses. The interaction of rheumatoid factor with biologically inactive soluble complexes to produce larger insoluble but active complexes has been previously described. ${ }^{11}$ We interpret our observation, where neutrophil activation occurred with incubation of inactive HAGG following preincubation with $\mathrm{mRF}$, as reflecting cross-linking by the $\mathrm{mRF}$ reagent. This would result in aggregates of large size.

Further indirect evidence that this modulation of neutrophil activation was related to an increase in aggregate size was observed in kinetic studies. The CL response curve showed a more significant increase in the slower second peak than the primary peak, suggesting that the increases may be due to increased phagocytic activity.
Preincubation of insoluble HAGG with polyclonal rheumatoid factor had no effect on activation of the neutrophil respiratory burst in the study of Turner $e t$ al. ${ }^{11}$ However, using an mRF reagent we observed a depression in the $\mathrm{CL}$ responses and have subsequently made similar observations using a polyclonal rheumatoid factor (unpublished observations). The kinetic studies showed a more significant depression of the immediate response than in the slower phagocytic response. The reason for this depression is unclear, but we postulate a masking of $\operatorname{IgG~Fc}$ by the highly specific $\mathrm{mRF}$.

An equivalence curve of preformed immune complexes provided a range of sizes of complexes varying from large insoluble complexes at equivalence to small soluble complexes in both antigen or antibody excess. The neutrophil activation profile of large insoluble complexes, with maximal stimulation occurring with complexes containing slight antigen excess, has been previously reported. ${ }^{12}$ Similar observations with neutrophil inhibitor studies have also been described. ${ }^{13}$ Our findings support the hypothesis that the immunochemical properties of immune complexes are of importance in determining whether they will cause direct activation on neutrophils.

The modulation of neutrophil $\mathrm{CL}$ activation by preincubation of $\mathrm{mRF}$ with preformed immune complexes or with HAGG was strikingly similar. Depression of the maximal neutrophil responses to large insoluble complexes and increased responses to inactive small soluble complexes were observed. These findings may suggest a useful biological role for rheumatoid factors whereby phagocytosis of circulating complexes is enhanced while oxygen-free radical production by neutrophils is depressed. Lamers $e t$ al. ${ }^{14}$ have also studied the influence of rheumatoid factors on the processing of DNA/anti-DNA complexes by neutrophils and suggest a similar role for rheumatoid factor.

Neutrophil activation by immune complexes leading to the release of oxygen-free radicals and lysosomal enzymes has been implicated in the immunopathogenesis of several immune-complexmediated diseases. The detection and characterisation of immune complexes capable of this direct neutrophil activation is therefore of clinical significance. We have demonstrated, using neutrophil $C L$, that complex size and antigen/antibody ratios are important properties of immune complexes in determining their ability to activate neutrophils. In addition we have demonstrated the modulation of this interaction by rheumatoid factor.

\footnotetext{
References

1 Starkebaum G, Stevens D L, Henry C, Gavin S E. Stimulation of human neutrophil chemiluminescence by soluble immune
} 
complexes and antibodies to neutrophils. J Lab Clin Med 1981; 98: 280-91.

2 Zubler R H, Nydegger V, Perrin L H, et al. Circulating and intra-articular immune complexes in patients with rheumatoid arthritis. J Clin Invest 1976; 57: 1038-319.

3 Johnston R B, Lehmeyer J E. Elaboration of toxic oxygen-by-products by neutrophils in a model of immune complex disease. J Clin Invest 1976; 57: 836-41.

4 Gale R, Bertouch J V, Bradley J, Roberts-Thomson P J. Direct activation of neutrophil chemiluminescence by rheumatoid sera and synovial fluid. Ann Rheum Dis 1983; 42: 158-62.

5 Roberts-Thomson P J, Bradley J. A nephelometric study of the reaction of monoclonal rheumatoid factor with heat aggregated gammaglobulin and sera from patients with immune complex diseases. Clin Exp Immunol 1979; 37: 408-15.

6 Cairns S A, London A, Mallick N P. The value of three immune complex assays in the management of systemic lupus erythematosus: an assessment of immune complex levels, size and immunochemical properties in relation to disease activity and manifestations. Clin Exp Immunol 1980; 40: 273-82.

7 Lamers M C, De Groot E R, Roos D. Phagocytosis and degradation of DNA-anti:DNA complexes by human phagocytes. Eur J Immunol 1981; 11: 764-8.

8 McCarthy D, Goddard D H, Pell B K, Holborow E J.
Intrinsically stable IgG aggregates. J Immunol Methods 1981; 41: 63-74.

9 Logfren S, Tarnvik A, Carlsson J. Influence of complement on the chemiluminescent response of human leukocytes to immune complex. Infect Immun 1980; 29: 335-41.

10 Goldstein I M, Roos D, Kaplan H B, Weissmann G. Complement and immunoglobulins stimulate superoxide production by human leukocytes independently of phagocytosis. J Clin Invest 1975; 56: 1155-63.

11 Turner R, Collins R, Stott K, Kaufmann J, Parker M, DeChatelet $\mathrm{L}$. Immunoglobulin $\mathrm{G}$ complex interactions with rheumatoid factor and neutrophils: ${ }^{51} \mathrm{CrCl}_{3}$ labelling and ${ }^{14} \mathrm{CO}_{2}$ hexose monophosphate shunt studies. $J$ Rheumatoid 1976; 3: 329-35.

12 Nydegger $\mathbf{R}$ M, Anner R M, Gerebtzoff A, Lambert P H, Miescher P A. Polymorphonuclear leukocyte stimulation by immune complexes. Assessment by nitroblue tetrazolium dye reduction. Eur J Immunol 1973; 3: 465-70.

13 Doll $\mathrm{N} \mathrm{J}$, Wilson $M \mathrm{R}$, Salvaggio $\mathrm{J}$ E. Inhibition of polymorphonuclear leukocyte chemiluminescence for detection of immune complexes in human sera. J Clin Invest 1980; 66: 457-64.

14 Lamers M C, de Groot E R, Roos D. Phagocytosis and degradation of DNA-antiDNA complexes by human phagocytes. Influence of theumatoid factors. $J$ Rheumatol 1981; 8: 880-888. 